\title{
Cardiac surgery practice during the COVID-19 outbreak: a regionwide survey
}

\author{
Luca Salvatore De Santo $^{1 \wedge}$, Antonino Salvatore Rubino ${ }^{1 \wedge}$, Michele Torella $^{1 \wedge}$, Denise Galbiati $^{1}$, \\ Gabriele Iannellii ${ }^{2}$, Severino Iesu ${ }^{3}$, Francesco Paolo Tritto ${ }^{4} \wedge$, Brenno Fiorani ${ }^{5}$, Luigi Chiariello ${ }^{6}$, \\ Antonio De Bellis ${ }^{7}$, Giuseppe Di Benedetto ${ }^{8}$, Carlo Zebele ${ }^{9}$, Marisa De Feo ${ }^{1 \wedge}$
}

${ }^{1}$ Cardiac Surgery Unit, Department of Translational Medical Sciences, University of Campania "Luigi Vanvitelli”, AORN dei Colli, Cardiac Surgery, Vincenzo Monaldi Hospital, Naples, Italy; ${ }^{2}$ Department of Advanced Biomedical Sciences, Cardiac Surgery Unit, University of Naples Federico II, Policlinico Federico II, Naples, Italy; ${ }^{3}$ Cardiac Surgery Unit, Azienda Ospedaliera San Giovanni di Dio e Ruggi D’Aragona, Salerno, Italy; ${ }^{4}$ Cardiac Surgery Unit, Azienda Ospedaliera Di Caserta - Sant'Anna e San Sebastiano, Caserta, Italy; ${ }^{5}$ Cardiac Surgery Unit, Azienda Ospedaliera San Giuseppe Moscati, Città Ospedaliera - Contrada Amoretta, Avellino, Italy; ${ }^{6}$ Cardiac Surgery Unit, Clinica Mediterranea, Naples, Italy; ${ }^{7}$ Cardiac Surgery Unit, Casa di Cura San Michele, Maddaloni, Caserta, Italy; ${ }^{8}$ Cardiac Surgery Unit, Pineta Grande Hospital, Castel Volturno, Caserta, Italy; ${ }^{9}$ Cardiac Surgery Unit, Casa di Cura Montevergine, Mercogliano, Avellino, Italy

Contributions: (I) Conception and design: LS De Santo, AS Rubino, M Torella; (II) Administrative support: None; (III) Provision of study materials or patients: D Galbiati, G Iannelli, S Iesu, FP Tritto, B Fiorani, L Chiariello, A De Bellis, G Di Benedetto, C Zebele; (IV) Collection and assembly of data: D Galbiati, G Iannelli, S Iesu, FP Tritto, B Fiorani, L Chiariello, A De Bellis, G Di Benedetto, C Zebele; (V) Data analysis and interpretation: AS Rubino; (VI) Manuscript writing: All authors; (VII) Final approval of manuscript: All authors.

Correspondence to: Antonino Salvatore Rubino, MD, PhD. Assistant Professor of Cardiac Surgery, Department of Translational Medical Sciences, University of Campania "Luigi Vanvitelli”, AORN dei Colli, Cardiac Surgery, Vincenzo Monaldi Hospital, Via Leonardo Bianchi 5, 80131 Naples, Italy. Email: antoninosalvatore.rubino@unicampania.it.

Background: Health systems worldwide have been overburdened by the "COVID-19 surge". Consequently, strategies to remodulate non-COVID medical and surgical care had to be developed. Knowledge of the impact of COVID surge on cardiac surgery practice is mainstem. Present study aims to evaluate the regional practice pattern during lockdown in Campania.

Methods: A multicenter regional observational 26-question survey was conducted, including all adult cardiac surgery units in Campania, Italy, to assess how surgical practice has changed during COVID-19 national lockdown.

Results: All centers adopted specific protocols for screening patients and personnel. A significant reduction in the number of dedicated intensive care unit (ICU) beds $(-30.0 \% \pm 38.1 \%$, range: $0-100 \%)$ and cardiac operating rooms $(-22.2 \% \pm 26.4 \%$, range: $0-50 \%)$ along with personnel relocation to other departments was disclosed (anesthesiologists $-5.8 \% \pm 11.1 \%$, range: $0-33.3 \%$; perfusionists $-5.6 \% \pm 16.7 \%$, range: $0-50 \%$; nurses $-4.8 \% \pm 13.2 \%$, range: $0-40 \%$; cardiologists $-3.2 \% \pm 9.5 \%$, range: $0-28.6 \%$ ). Cardiac surgeons were never reallocated to other services. Globally, we witnessed dramatically lower adult cardiac surgery case volumes (335 vs. 667 procedures, $\mathrm{P}<0.001$ ), as institutions and surgeons followed guidelines to curtail nonurgent operations.

Conclusions: This regional survey demonstrates major changes in practice as a response to the COVID-19 pandemic. In this respect, this experience might lead to the development of permanent systems-based plans for future pandemic and may effectively help policy decision making when prioritizing healthcare resource reallocation during and after the pandemic.

\footnotetext{
^ ORCID: Luca Salvatore De Santo, 0000-0002-8958-9063; Antonino Salvatore Rubino, 0000-0002-9399-7422; Michele Torella, 00000003-3853-3171; Gabriele Iannelli, 0000-0001-6464-9798; Francesco Paolo Tritto, 0000-0002-3869-8403; Marisa De Feo, 0000-0001$6331-3643$.
} 
Keywords: Cardiac surgery; COVID-19 pandemic; waiting list; healthcare resources; prioritization

Submitted Jun 24, 2020. Accepted for publication Oct 28, 2020.

doi: $10.21037 /$ jtd-20-2298

View this article at: http://dx.doi.org/10.21037/jtd-20-2298

\section{Introduction}

Health systems worldwide have been overburdened by the "COVID-19 surge" with an unprecedented demand for diagnostics and treatment. Italy has been the first European country affected by this pandemic, and the third, beyond the United Kingdom and Spain, for the severity of the outbreak. Despite a series of attempts to control the spread of the infection, the prevalence rose significantly and led to a nationwide lockdown on 9th March 2020 that ended up only on May 4th. To date (4th June 2020), the total number of assessed cases is 234,013 and, at least, 33,689 patients died. There has been substantial regional variation within the country, particularly extreme in the populous northeast. A critical shortage of personal protective equipment (PPE), intensive care unit (ICU) beds, and respiratory ventilators became dramatically evident. Resources had to be reallocated leaving many patients, especially those with other ailments, without proper care (1). Consequently, strategies to remodel non-COVID medical and surgical care had to be developed. As far as cardiac surgery is concerned recent authoritative papers have disclosed possible roadmaps and proposed regional system reorganization models (2-5). Knowledge of the impact of COVID surge on cardiac surgery practice is mainstem. Given the interregional variations in the severity of the surge and in the baseline differences in healthcare resources, the opportunity to quantify the experience and changes implemented across regionwide setting is relevant to guide policy decision making when prioritizing healthcare resource reallocation during and after the pandemic. Indeed, operating capacity during the COVID-19 recovery period (phase 2) will have a dramatic impact on the time to clear the deferred cases backlog with a potential on morbidity and mortality rates (6). Present study aims to evaluate the regional practice pattern during lockdown in Campania. We present the following article in accordance with the MDAR reporting checklist (available at http://dx.doi.org/10.21037/jtd-20-2298).

\section{Methods}

A multicenter regional observational 26-question survey was conducted, including all adult cardiac surgery units in Campania, Italy, to assess how surgical practice has changed during COVID-19 national lockdown. Patients $<18$ years old were excluded from the study.

Redistribution of dedicated healthcare resources was investigated. Modalities for screening of surgical candidates along with roots of active surveillance of healthcare professional were analyzed. Availability of appropriate PPE was also recorded. All consecutive patients referred for surgery during the lockdown were included. Number, urgency status, type of surgical procedures, along with patterns of referral and discharge, as well as length of hospitalization were collected. These parameters where compared to those pertaining the equivalent period of 2019 practice.

\section{Data collection}

Each center retrieved data from their internal records or from Department of Management. Fill-in case reports were submitted to the coordinating unit at Department of Translational Medical Sciences, University of Campania, Naples, which was in charge for data collection, data analysis and report.

The study was conducted in accordance with the Declaration of Helsinki (as revised in 2013). The study was approved by institutional committee board of Department of Translational Medical Sciences, University of Campania (registration number 7 obtained on the March 2nd, 2020). Informed consent was waived because the survey itself did not include any sensitive data.

\section{Statistical analysis}

Categorical variables are presented as count and percentage, 
continuous data as means \pm standard deviations. Data were compared with chi-square and paired-sample $t$-test. Statistical significance was set at an alpha level of 0.05. All analyses have been performed with IBM SPSS Statistic 24 for Mac OS.

\section{Results}

Among all 9 regional hospitals, 2 were academic (22.2\%), 3 public hospitals $(33.3 \%)$ and 4 private clinics (44.4\%). Five were hub hospitals for COVID-19 patients.

\section{Screening protocols and protective equipment availability}

All centers adopted specific protocols for triaging of patients admitted to the hospital. Naso- and oro-pharyngeal swabs were the most performed preoperative screening tests for surgical candidates ( 6 centers $66.6 \%$-swab only: 3 centers, swab plus serum tests: 3 centers). Rapid test was the preferred test in $2(22.2 \%)$, serum antibodies in $1(11.1 \%)$. Protocols of surveillance for healthcare professionals were implemented in all centers, ranging from self-assessment to body temperature, from swabs to rapid test to antibodies title or various combinations of them. Use of PPE was mandatory in all Centers and was sufficiently provided in 6 (66.6\%), whereas only temporarily inadequate in 3 (33.3\%). No intrahospital COVID outbreak was reported.

\section{Resource allocation}

Hospital services were redistributed to differentiate pathways for COVID-19 positive/suspect positive patients. Eight centers had dedicated area for triage (4 inside the ward; 4 outside the ward). Four centers had also an operative room dedicated to COVID-19 positive/suspect positive patients (in all cases inside the operating block). Seven centers had dedicated ICU beds available for patients with positive or on-going screening tests ( 5 inside the cardiac ICU; 2 in the general ICU).

Eight centers $(88.9 \%)$ experienced a reduction of resources during the lockdown. In particular, the ICUs were mostly involved, with an average bed reduction of $-30.0 \% \pm 38.1 \%$ beds (range: $0-100 \%$ ) compared to 2019 standards, followed by operative rooms $(-22.2 \% \pm 26.4 \%$, range: $0-50 \%)$ and wards $(-14.6 \% \pm 22.5 \%$, range: $0-50 \%)$. As far as professionals were considered, 4 centers experienced a reduction of personnel. In particular, the greater reallocation involved the anesthesiologists
$(-5.8 \% \pm 11.1 \%$, range: $0-33.3 \%)$, followed by perfusionists $(-5.6 \% \pm 16.7 \%$, range: $0-50 \%)$, nurses $(-4.8 \% \pm 13.2 \%$, range: $0-40 \%)$, and cardiologists $(-3.2 \% \pm 9.5 \%$, range: $0-28.6 \%)$. The staff of cardiac surgeons was never reallocated to other services in all centers. The admittance of relatives was forbidden in 6 centers and limited in 3 . When relatives were not allowed to visit patients, bulletins were provided by telephone in all cases ( 1 center also allowing consultation in dedicated spaces). In the other 3 centers, consultations with the referring physician were available in dedicated spaces before surgery, at the end of surgery and during the whole hospital stay until discharge.

\section{Referral, operative planning, and surgical volumes}

Patterns of referral significantly changed compared to the equivalent period of 2019 practice (Table 1). In particular, there was a significant reduction of patients admitted from the waiting lists $(21.3 \%$ during lockdown vs. $39.3 \%$ in the $2019)$ in favor of an increased referral by the emergency contact $(15.0 \%$ vs. $13.1 \%)$, in-hospital consultations (35.4\% vs. $28.8 \%)$ or peripheral hospital referral $(28.0 \%$ vs. $16.0 \%)$. The absolute distribution of the etiologies was similar between the two study periods, with acute coronary syndromes (ACS) and valvular heart diseases being the main diagnoses. A significant drop of surgery for chronic coronary artery diseases (CADs), along with a relative increase of acute aortic syndromes were also observed (Figure 1). Operative planning was regular only in 4 centers, whereas in the other 5 was impaired by resource allocation (mainly delayed diagnostics and reduced availability of blood substitutes). Accordingly, overall procedural volume was significantly reduced during lockdown compared to 2019 (335 vs. 667 procedures, $\mathrm{P}<0.001$ ). Notably, the percentage of elective surgery dropped significantly, in favor of urgent and emergency procedures (Tables 1,2). Details on the surgical procedures performed are reported in Figure 2.

\section{Length of hospitalization and patterns of discharge}

Four centers $(44.4 \%)$ reported an increase in the average postoperative length of stay compared to the equivalent period in $2019(17.3 \pm 4.6$ vs. $10.7 \pm 2.3$ days, $\mathrm{P}<0.001)$. During the lockdown, only 2 centers did not change their pattern of discharge, maintaining the usual standard of care. On the other hand, 6 centers preferably discharged home and 1 center $(11.1 \%)$ kept sending patients directly to a rehabilitation facility. 
Table 1 Details of procedures performed during lockdown, compared to the same period of 2019

\begin{tabular}{|c|c|c|c|}
\hline Details & Lockdown 2020 & 2019 & $P$ \\
\hline Elective, n (\%) & $96(28.7)$ & $478(71.7)$ & \\
\hline Urgent, n (\%) & $207(61.8)$ & $150(22.5)$ & \\
\hline Emergency, n (\%) & $19(5.7)$ & $26(3.9)$ & \\
\hline Referral, n (\%) & & & $<0.001$ \\
\hline Emergent surgery & $38(15.0)$ & $81(13.1)$ & \\
\hline Urgent surgery from inpatient & $90(35.4)$ & $178(28.8)$ & \\
\hline Urgent surgery from regional referral & $71(28.0)$ & $99(16.0)$ & \\
\hline Unknown & 0 & $17(2.7)$ & \\
\hline
\end{tabular}

*, Outpatients called from the center waiting list.

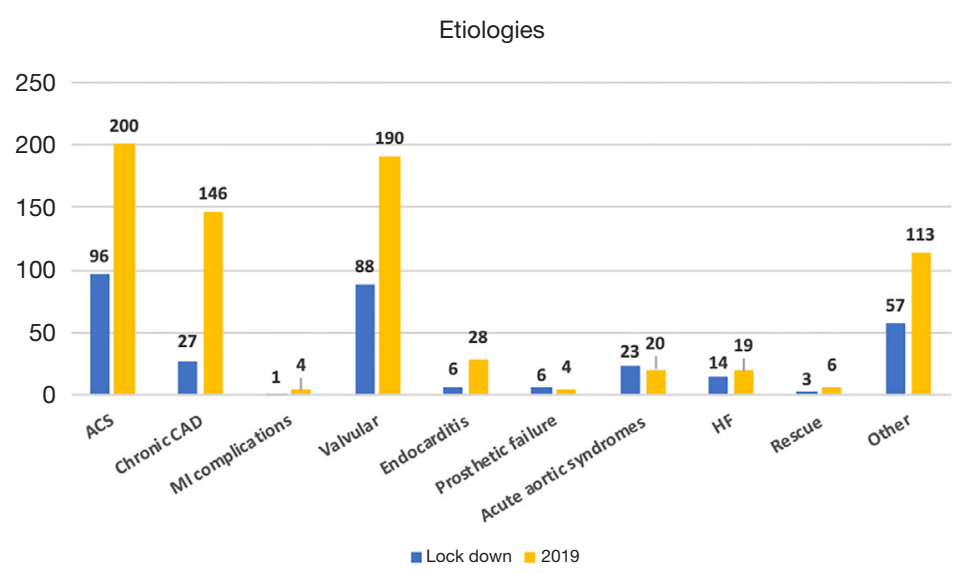

Figure 1 Distribution of etiologies between the two study periods. ACS, acute coronary syndromes; CAD, coronary artery disease; MI, myocardial infarction; HF, heart failure.

\section{Discussion}

The current pandemic has had an unprecedented impact on healthcare. We herein quantified the experience and changes implemented across adult cardiac surgery centers within Campania region. Boosting and optimizing the capacity of health systems to respond to the surge has been one of the major challenges. Policy responses had to be organized along three key priorities: staff, supplies, and space. A significant reduction in the number of dedicated cardiac operating rooms and ICU beds along with personnel relocation to other departments was indeed disclosed. As these systems-based changes were evolving, guidelines with adequate specificity to address the complexity of decision-making for safely and effectively performing and/ or deferring cardiac surgery were unavailable or, under development, at best (2-5). As a result, many hospital systems in combination with their heart teams developed program-specific policies. As an example, a recent authoritative paper described such reorganization pathways and management algorithms in Lombardy region (7). As a matter of fact, in Campania, preoperative screening 
Table 2 Breakdown of type of procedures by emergency status

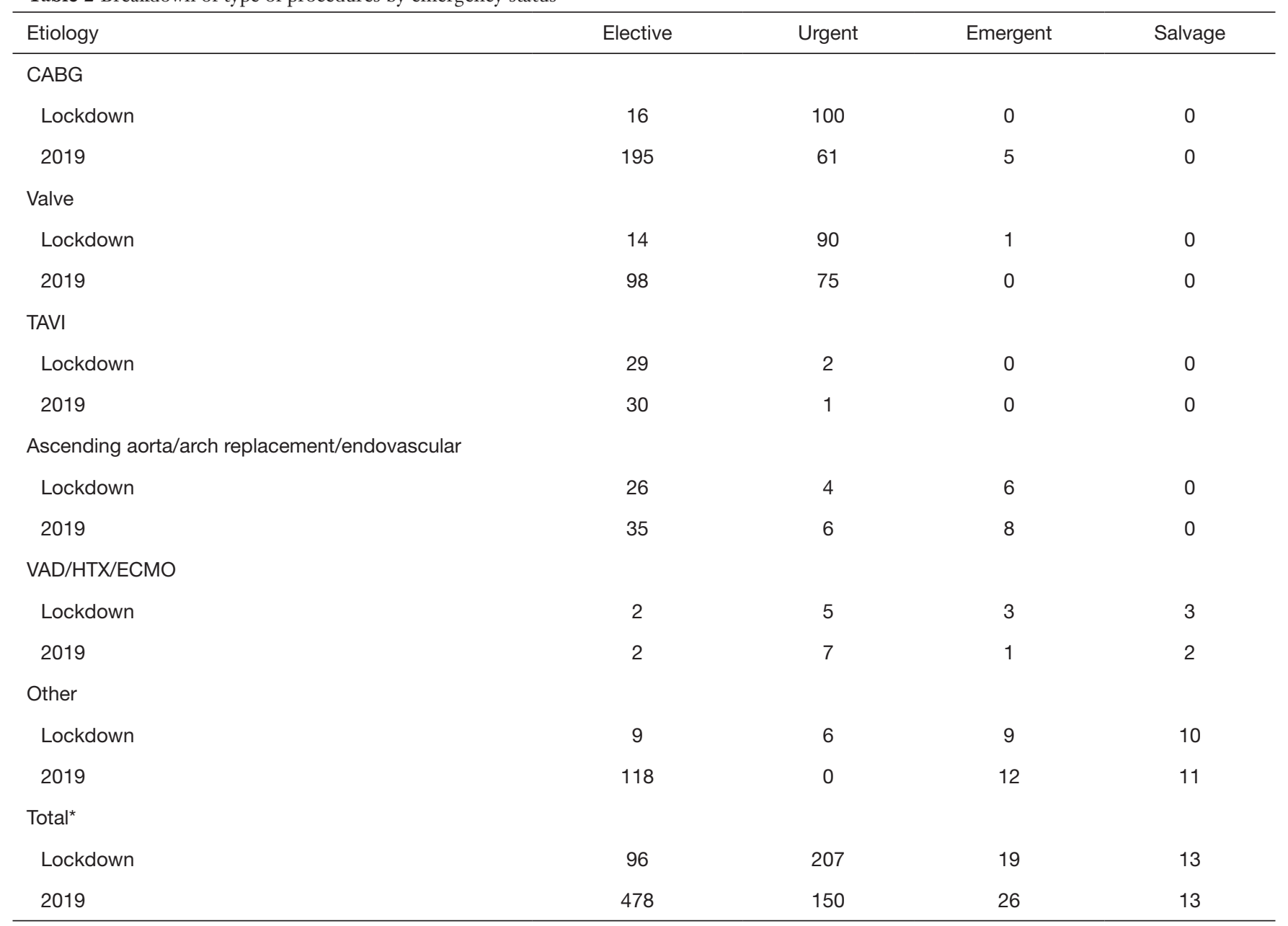

*, As reported in Table 1. Valve: repair, replacement. CABG, coronary artery bypass graft; TAVI, transcatheter aortic valve implantation; VAD, ventricular assist device; HTX, heart transplantation; ECMO, extracorporeal membrane oxygenation.

for COVID-19 disease status and algorithms of active surveillance for healthcare providers significantly differed from one institution to another. Similarly, modalities for access of patients' relatives to surgical wards, length of postoperative hospitalization and patterns of discharge widely varied. Notably, hospital LOS and the patterns of discharge were also affected by the COVID-19 pandemic. The competition for diagnostics, the scarcity of blood products, the need to maximize patient health status before discharge in times of limited access to cardiac surgery outpatient clinics, along with the scarcity of rehabilitation facilities, are among the factors which synergistically contributed to these practice changes. Usually, resource availability is not factored in the decision-making process and the choices of an individual clinical or surgical case (8).
PPE has been an important and emotive subject during the current pandemic: appropriate use significantly reduces risk of viral transmission, but a worldwide limited availability has become critically evident (9). Shortage of PPE has been experienced in variable degrees in the Campania surgical centers, with one-third suffering from a critical scarcity. Nevertheless, the satisfactory freedom from infection of cardiac surgery health care workers and lack of intrahospital COVID-19 outbreaks testifies for the efficacy of such "homemade" management pathways and judicious deployment of limited resources. Two data might be worth a comment. First, coronary surgery for ACS significantly and worryingly dropped during the lockdown. This unexpected pattern mirrors that reported by an Italian nationwide cardiologists' survey reporting a dramatic reduction in 
Surgical procedures

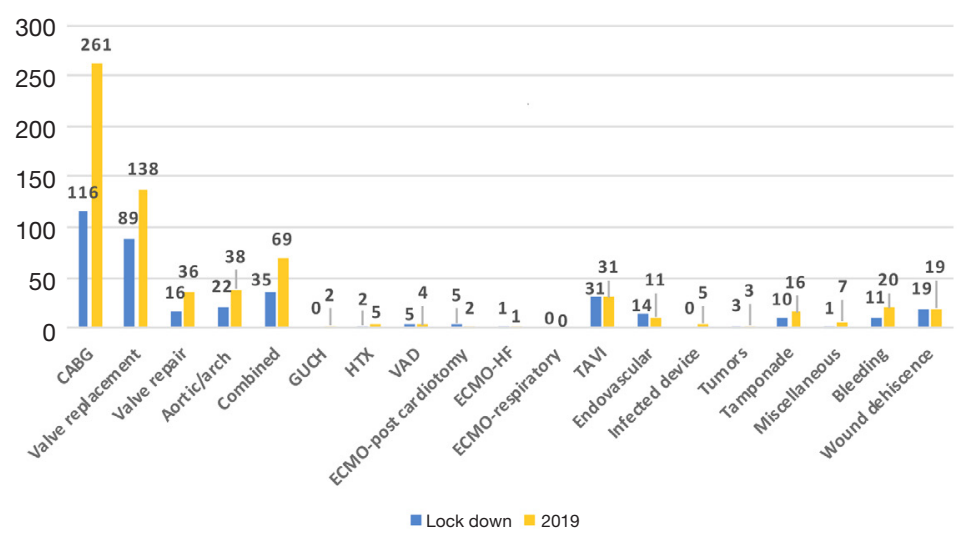

Figure 2 Specific surgical volumes during the two study periods. CABG, coronary artery bypass graft; GUCH, grown up congenital heart disease; HTX, heart transplantation; VAD, ventricular assist device; ECMO, extracorporeal membrane oxygenation; TAVI, transcatheter aortic valve implantation.

admissions for acute myocardial infarction (MI) during the lockdown and an escalation of inherent mortality and complication rates (10). Second, the number of transcatheter aortic valve implantations (TAVIs) appeared unaffected by the COVID-19 surge. Again, this pattern is homogeneous to that reported by a contemporary survey and testifies of modified treatment algorithm for severe aortic stenosis in times of limited healthcare resources $(11,12)$. Globally, we witnessed dramatically lower adult cardiac surgery case volumes, as institutions and surgeons followed guidelines to curtail non-urgent operations. The median reduction in cardiac surgery case volume was 53.5\% (IQR: 39.6-81.9\%). Such a pattern is consistent with recently published international surveys $(11,12)$. Most centers restricted cardiac surgery activity to urgent/emergent cases; 6 centers had cancelled elective cases. Such a decrease has had obvious positive effects. Indeed, it spared limited hospital resources, prevented in hospital spreading of COVID-19 and limited unnecessary risk of operating on asymptomatic patients within the infection incubation period. The drawbacks of such practice patterns are less clear now, but the outlook is not optimistic. Indeed, contemporary mortality rate while waiting for elective cardiac surgical procedures are still relevant. As an example, patients needing surgical or percutaneous aortic valve replacement experience mortality rates up to $3.7 \%$ at 1 month and $11.6 \%$ at 6 months (13). In patients listed for surgical myocardial revascularization waiting list mortality averages $6 \%$ per month, with risk increasing $11 \%$ per month, with a concurrent significant incidence of acute MI (14). More, increased mortality has been previously reported by healthcare systems in the aftermath of natural disasters $(15,16)$. As a matter of fact, clearance of backlog cardiac surgical cases will be complicated by exhaustion of supplies and resources from the pandemic, and competition with similar needs by other medical and surgical subspecialties. In this respect, data reported in this paper may effectively help policy decision making when prioritizing healthcare resource reallocation after the pandemic (17). All in all, the pattern described herein adds to the concerning observation that excess non-COVID-19 mortality may lately overcome that directly related to COVID-19 infections.

\section{Study limitations}

Present study suffers from several limitations. First, it is a snapshot of a rapidly evolving situation within a context of limited resources and unsettled practice guidelines during an unprecedented pandemic. Second, the survey design implies an inherently potential for subjectivity. Third, we have assumed that historical rates of surgery might provide a valuable benchmark to quantify surgical backlog and thus post surge need for cardiac surgery escalation.

\section{Conclusions}

In conclusion, this regional survey demonstrates major changes in practice as a response to the COVID-19 
pandemic. The cardiac surgical network responded expeditiously and effectively despite severe shortage of healthcare resources in terms of space, staff, PPE and despite the absence of available specific guidelines. In this respect, this experience might lead to the development of permanent systems-based plans for future pandemic. Finally, present survey quantifies at large the backlog of cardiac surgical procedures. These data may effectively help policy decision making when prioritizing healthcare resource reallocation after the pandemic.

\section{Acknowledgments}

Funding: This study has been partially supported by an unrestricted grant (program VALERE) from the University of Campania "Luigi Vanvitelli".

\section{Footnote}

Reporting Checklist: The authors have completed the MDAR reporting checklist. Available at http://dx.doi.org/10.21037/ jtd-20-2298

Data Sharing Statement: Available at http://dx.doi. org/10.21037/jtd-20-2298

Conflicts of Interest: All authors have completed the ICMJE uniform disclosure form (available at http://dx.doi. org/10.21037/jtd-20-2298). The authors have no conflicts of interest to declare.

Ethical Statement: The authors are accountable for all aspects of the work in ensuring that questions related to the accuracy or integrity of any part of the work are appropriately investigated and resolved. The study was conducted in accordance with the Declaration of Helsinki (as revised in 2013). The study was approved by institutional committee board of Department of Translational Medical Sciences, University of Campania (registration number 7 obtained on the March 2nd, 2020). Informed consent was waived because the survey itself did not include any sensitive data.

Open Access Statement: This is an Open Access article distributed in accordance with the Creative Commons Attribution-NonCommercial-NoDerivs 4.0 International License (CC BY-NC-ND 4.0), which permits the noncommercial replication and distribution of the article with the strict proviso that no changes or edits are made and the original work is properly cited (including links to both the formal publication through the relevant DOI and the license). See: https://creativecommons.org/licenses/by-nc-nd/4.0/.

\section{References}

1. Rosenbaum L. Facing Covid-19 in Italy - ethics, logistics, and therapeutics on the epidemic's front line. $\mathrm{N} \mathrm{Engl} \mathrm{J}$ Med 2020;382:1873-5.

2. Engelman DT, Lother S, George I, et al. Adult cardiac surgery and the COVID-19 pandemic: aggressive infection mitigation strategies are necessary in the operating room and surgical recovery. Ann Thorac Surg 2020;110:707-11.

3. Hassan A, Arora RC, Lother SA, et al. Ramping up the delivery of cardiac surgery during the COVID-19 pandemic: a guidance statement from the Canadian Society of Cardiac Surgeons. Can J Cardiol 2020;36:1139-43.

4. Haft JW, Atluri P, Ailawadi G, et al. Adult cardiac surgery during the COVID-19 pandemic: a tiered patient triage guidance statement. Ann Thorac Surg 2020;110:697-700.

5. Bonalumi G, di Mauro M, Garatti A, et al. The COVID-19 outbreak and its impact on hospitals in Italy: the model of cardiac surgery. Eur J Cardiothorac Surg 2020;57:1025-8.

6. Parolari A, di Mauro M, Bonalumi G, et al. Safety for all: coronavirus disease 2019 pandemic and cardiac surgery: a roadmap to 'phase' 2. Eur J Cardiothorac Surg 2020;58:213-6.

7. Belluschi I, De Bonis M, Alfieri O, et al. First reorganization in Europe of a regional cardiac surgery system to deal with the coronavirus-2019 pandemic. Eur J Cardiothorac Surg 2020;58:25-9.

8. Emanuel EJ, Persad G, Upshur R, et al. Fair allocation of scarce medical resources in the time of Covid-19. N Engl J Med 2020;382:2049-55.

9. Mandrola J. CoViD-19 and PPE: some of us will die because of the shortage. Recenti Prog Med 2020;111:183.

10. De Rosa S, Spaccarotella C, Basso C, et al. Reduction of hospitalizations for myocardial infarction in Italy in the COVID-19 era. Eur Heart J 2020;41:2083-8.

11. Gaudino M, Chikwe J, Hameed I, et al. Response of cardiac surgery units to COVID-19: an internationallybased quantitative survey. Circulation 2020;142:300-2.

12. Seese L, Aranda-Michel E, Sultan I, et al. Programmatic responses to the coronavirus pandemic: a survey of 502 cardiac surgeons. Ann Thorac Surg 2020;110:761-3.

13. Malaisrie SC, McDonald E, Kruse J, et al. Mortality while 
waiting for aortic valve replacement. Ann Thorac Surg 2014;98:1564-70; discussion 1570-1.

14. Seddon ME, French JK, Amos DJ, et al. Waiting times and prioritization for coronary artery bypass surgery in New Zealand. Heart 1999;81:586-92.

15. Lee EE, Stewart B, Zha YA, et al. Surgical care required for populations affected by climate-related natural disasters: a global estimation. PLoS Curr 2016;8:ecurrents. dis.e601960a8cd66c3083d160877abfdde4.

16. Chan EY, Sondorp E. Medical interventions following natural disasters: missing out on chronic medical needs. Asia Pac J Public Health 2007;19 Spec No:45-51.

17. Salenger R, Etchill EW, Ad N, et al. The surge after the surge: cardiac surgery post-COVID-19. Ann Thorac Surg 2020;110:2020-5.
Cite this article as: De Santo LS, Rubino AS, Torella M, Galbiati D, Iannelli G, Iesu S, Tritto FP, Fiorani B, Chiariello L, De Bellis A, Di Benedetto G, Zebele C, De Feo M. Cardiac surgery practice during the COVID-19 outbreak: a regionwide survey. J Thorac Dis 2021;13(1):125-132. doi: 10.21037/jtd-202298 